L. Bannon, I. Wagner, C. Gutwin, R. Harper, and K. Schmidt (eds.). ECSCW'07: Proceedings of the Tenth European Conference on Computer Supported Cooperative Work, 24-28 September 2007, Limerick, Ireland

\title{
Behaviours and Preferences when Coordinating Mediated Interruptions: Social and System influence
}

\author{
Natalia Romero ${ }^{1}$, Agnieszka Matysiak Szóstek ${ }^{1}$, \\ Maurits Kaptein ${ }^{2}$ and Panos Markopoulos ${ }^{1}$ \\ ${ }^{1}$ Industrial Design, Eindhoven University of Technology, The Netherlands \\ ${ }^{2}$ USI Programme, Eindhoven University of Technology, The Netherlands \\ \{n.a.romero, a.matysiak, m.c.kaptein, p.markopoulos\} tue.nl
}

\begin{abstract}
There is a growing interest in technologies for supporting individuals to manage their accessibility for interruptions. The applicability of these technologies is likely to be influenced by social relationships between people. This paper describes an experiment that examines interplay between a working relationship of an interruptor and an interruptee and two different system approaches to handle interruptions. We tested how system behaviour and the social relationship between the actors influence their interruption behaviours. Our results are consistent with prior research on the importance of relational benefit to understanding interruption. We found that interruptors were far more likely to be considerate of interruptees' activities, when they both shared a common goal. We have extended those findings by showing that interruptees display similar behaviours to those presented by interruptors. The results regarding the systems' influence show a clear trend towards the positive effect of the Automatic system on peoples' interruption behaviours which is based on: (i) visible interruption costs, (ii) social tension and (iii) system preference. We think that the results of this experiment translated into design implications can prove helpful in informing the design of computer-mediated solutions supporting interruption handling.
\end{abstract}

\section{Introduction}

Informal communication, both collocated and distributed, appears to be one of the most successful communication channels in nowadays offices (Kraut, Fish et al. 
1990; Nardi, Whittaker et al. 2000; Nardi and Whittaker 2001). Such communication allows for rapid feedback, sharing local context, spontaneous conversations and referencing common depictions or values (Olson and Olson 2000). But there is a cost to it, interruptions. Nardi and Whittaker (2001), and Kakihara et al (2004) noted an asymmetry in control of interruptions between an interruptor and an interruptee that 'arises because while initiators benefit from rapid feedback, the recipients are forced to respond to the initiator agenda'. To deal with this visible inequity in control over interactive attempts, awareness systems have been proposed as mechanisms to support interruption negotiation (Dourish and Bly 1992; Nichols, Wobbrock et al. 2002; Begole, Matsakis et al. 2004; Wiberg and Whittaker 2005). However, empirical evaluations of those systems have shown that, although they positively influence the behavioral patterns of interruptors, they do not prevent interruptions from occurring at wrong moments (Fogarty, Hudson et al. 2005). Such findings indicate that the relative behaviour of two interruption actors is not only determined by the existence of an awareness system but is likely to be influenced by other factors ranging from individual (McFarlane and Latorella 2002; González and Mark 2004; Bailey, Konstan et al. 2005) to social (Perlow and Weeks 2002; Jett and George 2003; Patil and Lai 2005) and to technical aspects (Cheverst, Dix et al. 2005; Wiberg and Whittaker 2005). A better understanding of the dependencies between social and technological influences on interruption behaviours for both interruption actors can prove helpful in informing the design of computer-mediated solutions supporting interruption handling.

This paper describes an experimental study evaluating the influence of two factors on the interruption behaviour of interruptors and interruptees; we wanted to test the impact upon the actors' behaviour caused by: (i) whether they share a common goal or not and (ii) whether the awareness system filters incoming interruptions or not. Finally, we wanted to assess how behavioural change enforced by automatic interruptions' filtering is perceived in terms of user preferences.

\section{Related Work}

A variety of behaviours in handling interruptions have been previously noted (Altman 1975; Sproull 1984; Jett and George 2003; González and Mark 2004; Minassian, Muller et al. 2004). In a face-to-face situation, when initiating an interruption the interruptor usually decides whether to interrupt or not by assessing the interruptee's availability status through verbal and non-verbal clues produced by the interruptee him/herself (e.g., does one appear stressed or relaxed) and through signals gathered from the environment (e.g., is one present or absent?) (Sproull 1984; Kendon 1990; Hudson, Christensen et al. 2002; McFarlane and Latorella 2002). The decision whether to continue or to withdraw from the interruption may be further based on the nature of its subject. The interruptor may de- 
cide to abandon a trivial question if the interruptee appears busy but may be less considerate about potential costs to the interruptee when dealing with an issue of greater importance or urgency.

Once the interruption has been initiated, the interruptee has a choice of how to deal with an incoming communicative attempt. (S)he can choose between immediately handling, postponing or rejecting an interruption and also between providing a comprehensive or a partial answer (Goffman 1967; Clark 1996). An adequate behaviour is often motivated by the social and professional relationship between the actors (Kendon 1990; Patil and Lai 2005). It is also contingent upon other aspects such as an interruptee's own time-pressure or the next activity planned (Hudson, Christensen et al. 2002; Adamczyk and Bailey 2004; Bailey, Konstan et al. 2005; Gonzales and Mark 2005).

In the case of mediated interruption handling, two approaches can be contrasted: an automatic and a manual approach. In the automatic approach, the system takes a role of an interruption mediator so that both actors fully rely on its performance. Begole and Tang (2003) explored the feasibility of automatic availability inference based on activity monitoring. Another example of automated availability management systems are Personal Reachability Management Systems (PRMS) (Reichenbach, Damker et al. 1997). The benefits of PRMS relate to minimizing interruptees' effort when dealing with undesired communicative attempts by shifting effort upon the interruptor. Processing an interruption request is automated and is based on what the interruptor has specified as the context of the communication attempt and what the interruptee has pre-defined as criteria for interaction agreement. Regarding the manual approach, a system such as Push-to-Talk (Nardi, Whittaker et al. 2000) implements a set of outeraction mechanisms that allow users to manually coordinate their availability without interfering with the lightweight of the communication protocol. With their system NEGOTIATOR, Whitaker and Wiberg (2005) have shown how manual availability management might create social tension for the interacting parties.

Apart from the benefits of each system there are also costs associated to illtimed interruptions as well as effort to provide relevant context for reachability management. McFarlane (2002) experimentally compared different ways for coordinating interruptions in a computer-based multitasking context. Experiment subjects were asked to play a 'Jumpers Game' as their primary task, in which they had to save virtual game characters jumping from a building. While playing this game they were frequently interrupted by another task. McFarlane noted that participants' performance improved after they were allowed to control their interruptions by choosing the right moment for them to occur. The author concluded that in order to support mediated interruptions there is a need for tools that allow for assessing and announcing appropriate interruption moments.

Arguably, both automatic and manual approach can prove useful in different social relationships. Dabbish and Kraut (2004) extended McFarlane's experiment 
and investigated the use of awareness displays as instruments for supporting interruption coordination. They examined how awareness displays influence the choice of the interruption moment, how sharing a common goal increases their success ratio and, how the richness of presented information affects the interruption handling behaviour. They too used the 'Jumpers Game' as a primary task for the interruptee and introduced an 'Image Guessing' task for the interruptor. To complete their task successfully interruptors frequently needed help from their assigned interruptees. The results of this experiment showed that if the interacting parties share a common goal (Clark 1996), interruptors are more likely to display altruistic behaviour towards the interruptee: they will be more prone to assess interruptee's availability (Begole, Tang et al. 2003; Gonzalez and Mark 2004) and time-pressure (Adamczyk and Bailey 2004; Bailey, Konstan et al. 2005) before initiating the interruption.

The experiment by Dabbish and Kraut suggests that in a shared-goal situation an awareness display may, indeed, be an appropriate and sufficient stimulus for evoking altruistic interruption behaviour upon interruptors. However the experiment has also shown that in a non-shared-goal situation interruptors were likely to display somewhat individualistic behaviour: they were prone to interrupt whenever they were in need for help without paying attention to the interruptee's availability status. Considering that many interruptions in an actual working context arise not only from the team members sharing the same goal but also by other individuals, it seems reasonable to conjecture that a system assisting interruptions in a non-shared-goal situation cannot entirely rely on the awareness display and should be allowed to assess the interruption moment.

A number of interesting questions arise from the experiment of Dabbish and Kraut. Their study examined behaviours only for interruptors; it is also interesting to examine how the interruptees' behaviour is influenced by their relation with the interruptor. The authors contrasted two social relationships between the interruption actors: a team and an independent condition. The team condition was defined as: "being in group with another person and having outcome interdependence"; while the independent condition described a situation, in which: "the interruptors were rewarded exclusively on their own performance". This distinction results in an effective experimental manipulation, but is arguably not representative of the interruptions concerning office workers. Clearly, while the Team (shared-goal) condition is very characteristic for the office environment, the Independent condition is fairly rare for workers who are not directly dealing with customers or the general public. A more common source of interruptions for them is from people working for the same organizational unit (department or sub-department) though not on the same project (so therefore not sharing goals) (Chrysanthis, Stemple et al. 1990; Patil and Lai 2005). In line with Chrysanthis et al. (1990) a Group is defined as: "In the group, people can perform their tasks concurrently and independently, while interacting cooperatively to achieve own objectives". This social 
relationship is furthermore shaped by the existence of social reciprocation as defined by Perlow and Weeks (2002): “...the likelihood of receiving an interruption from the interruptee in the near future". Thus our experiment concerns a "team condition' as defined by Dabbish and Kraut and a 'group condition' as defined by Chrysanthis et al. (1990) and Perlow (2002).

Finally, next to the social relationship between actors, our experiment also compares the manual and automatic approach to handle interruptions. The automatic system manages availability of the interruptee (Reichenbach, Damker et al. 1997) by filtering the flow of interruptions, while the manual system provides participants full control over their interruptions (Nardi, Whittaker et al. 2000). We examine the impact of these two system types on the behaviour of interruption actors in the two social conditions described above. We add to current literature with an experimental assessment of how differences between the two system types and the social relationship impact the behaviours of the interruption actors.

\section{Experiment Description}

Our experiment had a two-fold objective. Firstly, we aimed to assess if the presence of a shared goal equally motivates interruptors and interruptees to display altruistic behaviours when dealing with interruptions. Secondly, we wanted to test the effect of an Automatic system to motivate more altruistic behaviours in the case of an absence of a shared goal between the interruption actors.

For the purposes of this experiment we have implemented two systems for interruption management: a Manual and an Automatic system (which are described in more detail below). The common structure of the two systems was defined so that neither system intervenes with the interruptor's decision to initiate the interruption and so that both systems provide their users with an abstract awareness display representing the status of the interruptee (Dabbish and Kraut 2004). The difference between the systems rests in the way they deal with incoming interruptions. The Automatic system filters interruptions that are ill-timed according to the ratio between the number of tasks to be performed by the interruptee and the time left to do so; it also automatically notifies the interruptor that his/her interruption has been rejected. The Manual system allows all interruptions to get through to the interruptee, so that the interruptee has to decide whether to accept or reject each interruption request.

As in the experiments discussed above, our set-up aimed to create 'an abstract help-seeking situation, in which two parties are collaborating' (Dabbish and Kraut 2004). In our experiment the two parties are: an Asker seeking help and a Helper who is engaged in an own task. We provided both actors with an abstract awareness display presenting them with the status of the Helper. Askers can choose the interruption moment and can also choose which out of a fixed set of 
questions to ask. Helpers can choose to answer immediately or reject the interruption, and they can also vary the quality of their responses.

\section{Definitions and Hypotheses}

We distinguish four interruption behaviours, two time-related behaviours and two content- related behaviours. Each behaviour pertains both to interruptions by Askers and to reactions by Helpers and has an altruistic or individualistic connotation (see: Table I).

\section{ASKER}

\section{HELPER}

Altruistic and individualistic behaviours that are time-related

Timely interruption: Asker's altruistic behaviour to initiate an interruption when the awareness display shows Helper's low timepressure.

Untimely interruption: Asker's individualistic behaviour to initiate an interruption when the awareness display shows Helper's high time-pressure.
Timely reaction: Helper's altruistic behaviour to immediately accept an incoming interruption.

Untimely reaction: Helper's individualistic behaviour to immediately reject an incoming interruption.

Altruistic and individualistic behaviours that are content-related

High-value question: Asker's altruistic behaviour to initiate an interruption with a high score associated to its content.

Low-value question: Asker's individualistic behaviour to initiate an interruption with a low score associated to its content.
High-value response: Helper's altruistic behaviour to provide response with a high value associated to its content.

Low-value response: Helper's individualistic behaviour to provide response with a low value associated to its content.

Table I. Askers' and Helpers' time and content-related behaviours used as dependent variables in the experiment

We expect to find that interruptors and interruptees who share a common goal (Team) will display more altruistic behaviours when dealing with interruptions compared to those who do not share a common goal. For interruptors this means matching the interruption moment with the interruptee's availability status and interrupting with high-value questions. We also expect that interruptees in most cases will be willing to accept incoming interruptions and put effort in providing interruptors with a thorough, high-value response. Such behaviours will remain consistent disregarding the system the Team uses, so the Team members will show similar behaviour in both the Manual and the Automatic system. We assume that the Manual system will expose the altruistic behaviours of actors sharing a 
common goal and individualistic behaviours of those, who do not share a common goal. Interruptors who do not share a common goal will interrupt at all times without being concerned about the interruptee's availability status. Also interruptees will be willing to accept incoming interruptions only when they perform well and do not experience time-pressure imposed by their own task. Interruptees, in any case, will not be willing to put effort to sustain the quality of their answers.

Furthermore, we believe that the Automatic system will influence the behaviours of people who do not share a common goal, so they will change their behaviours comparing to those presented in the Manual system. As shown by related literature (McFarlane 2002; Bailey, Konstan et al. 2005) interruptions produce negative consequences if they occur at times when the interruptee might experience time-pressure, anxiety and annoyance related to his/her primary task. So, we introduce an Automatic system that monitors the interruptee's performance and automatically rejects interruptions occurring whenever the interruptee may experience time-pressure related to his/her primary task (so interruptions are allowed only when the interruptee performs well). We believe that such an additional filtering will encourage interruptors in the Group condition to pay attention to the interruptee's availability status and try to time their interruptions better. We also think that interruptees in the Group condition will be more willing to accept interruptions and more considerate about providing comprehensive response if interruptions appear at right moments. To test these expectations we have formulated three hypotheses, which are introduced below.

\section{Hypothesis 1}

Both Helpers and Askers show more altruistic interruption behaviours in the Team condition than in the Group condition. We expect H1 to hold for both time and content-related behaviours.

\section{Hypothesis 2}

Both Helpers and Askers show more altruistic interruption behaviours when using the Automatic system than when using the Manual system. We expect $\mathrm{H} 2$ to hold for both time and content-related behaviours.

\section{Hypothesis 3}

The positive effect of the system-type, thus using the Automatic system on interruption behaviour is the strongest in the Group condition. We expect $\mathrm{H} 3$ to hold for both Group-Helpers and Group-Askers, and to apply for time as well as content-related behaviours.

\section{Participants}

A number of 35 males and 25 females participated in the experiment $(41-20$ to $30,17-30$ to $40,1-40$ to 50 and $1-50$ to 60 of age). 25 participants work in academia, 7 in industry, 26 were students and 2 were unemployed. They present 
different educational backgrounds: technical (18), design (18), psychology (3), economics (7) and others (8). Their educational level varies between: undergraduate (20), graduate (28) and PhD (12). Participants presented various nationalities and all were non-native English speakers. Most participants (42) reported having more than 2 years of experience using English on daily basis; the rest of the participants reported an experience between 1 and 2 years. All except one assigned pair were complete strangers to their partners in the game. In the familiar case the pair reported to be acquainted but had not worked together ever before or were they in any way professionally or socially linked.

\section{Design}

The experiment was a $2 \times 2$ mixed-subject design. The within subject factor was the system condition, which offered (i) a manual or (ii) an automatic approach to handling interruptions. This condition was randomized to avoid an order effect. The between subjects factor was the social condition, which identified two social relationships: the Team condition representing people sharing a common goal and the Group condition representing those who did not share a common goal but assumed social reciprocation.

\section{Procedure}

The experiment was conducted by the two first authors on the premises of the Eindhoven University of Technology and took form of a game, in which one Asker and one Helper won a prize of 25 euros each. Participants were divided in pairs and randomly assigned to their roles, and to the Team or Group condition. The players of each pair were placed in separate rooms so that they could not interact with each other in any way. Each pair played two rounds of the game: one using the Automatic and another using the Manual system (the order was randomised). The game began with an exploration phase, during which both players could become acquainted with the screens and controls. During the actual game, each round lasted 10 minutes. At the end of the second round a focus group was conducted.

In the Team condition, each Asker-Helper pair competed against other pairs; their scores were summed up and the best pair would win the prize. In the Group condition each Asker and each Helper competed individually with other Askers and Helpers; their individual scores were summed up, and the best Asker and the best Helper would win the prize. To create a feeling of a social reciprocation (Perlow and Weeks 2002) participants in both conditions were told that there would be a second phase of the game, in which they would swap their roles of Askers and Helpers.

Both Automatic and Manual system provided participants with an abstract awareness display (Dabbish and Kraut 2004) constructed out of two progress 
bars: the task bar that represents the progress of the Helper's task and the time bar that shows how much time was left for him/her to finish answering each question (see: Figure 1).
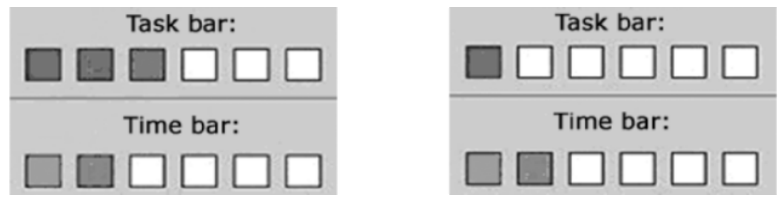

Figure 1. Left: the awareness display representing a timely interruption - the task bar is ahead of the time bar meaning that the Helper advances with the task and experiences low time-pressure; Right: the awareness display representing an untimely interruption - the time bar is ahead of the task bar meaning that the Helper stays behind the task and experiences high time-pressure.

Besides providing this information, neither system interfered with the Asker's decision to interrupt. The Automatic system filters the occurrence of an untimely interruption when Helper's task progress stayed behind his/her time progress at the moment the interruption was initiated. Any other interruption was interpreted as a timely interruption.

\section{Asker's Game}

The Asker receives an article divided in paragraphs, with 4 missing words per paragraph. (S)he has to fill in those missing words scoring points for each correct answer entered. The correct answer has to be chosen from a list of synonyms. Different words have different number of synonyms to choose from: some have one synonym and one correct word while others have four synonyms and one correct word to choose from. The word with one synonym and one correct word scores 2 points, while a word with four synonyms and one correct word scores 5 points. The Asker can confirm the chosen word with an assigned Helper who has access to the complete article, but who is busy playing another game. The Asker can check Helper's progress by recalling the awareness display.

Figure 2 shows the Asker's screen that is divided in two areas. The lower area contains the consecutive paragraphs of the article with missing words and a form to enter the chosen answers, with a 'Next' button to submit the words and move to the next paragraph. The upper area contains (from left to right) a form for sending questions, a timer and two buttons: the 'Progress Display' and the 'Option Display'. The 'Send Question' form is constructed out of a list with four numbers that represent the four lines containing missing words and a text field to enter the chosen word. The Asker sends a question by pressing the 'Ask Helper' button. The reaction of the Helper is shown at the same place on the screen and can be removed by using the 'Close' button. The timer counts down the time for each round in minutes except the last minute, which is counted in seconds. The 'Option 
Display' button activates for 10 seconds the list with synonyms of all missing words. The 'Progress Display' activates for 10 seconds the awareness display of the Helper's task and time progress. The task bar represents the task progression with each block representing one of the 6 items to be filled in by the Helper and the time bar representing time progression with each block representing 10 elapsed seconds of each Helper's question (see: Figure 1). The awareness display is updated every 10 seconds and reset once the Helper receives a new question.

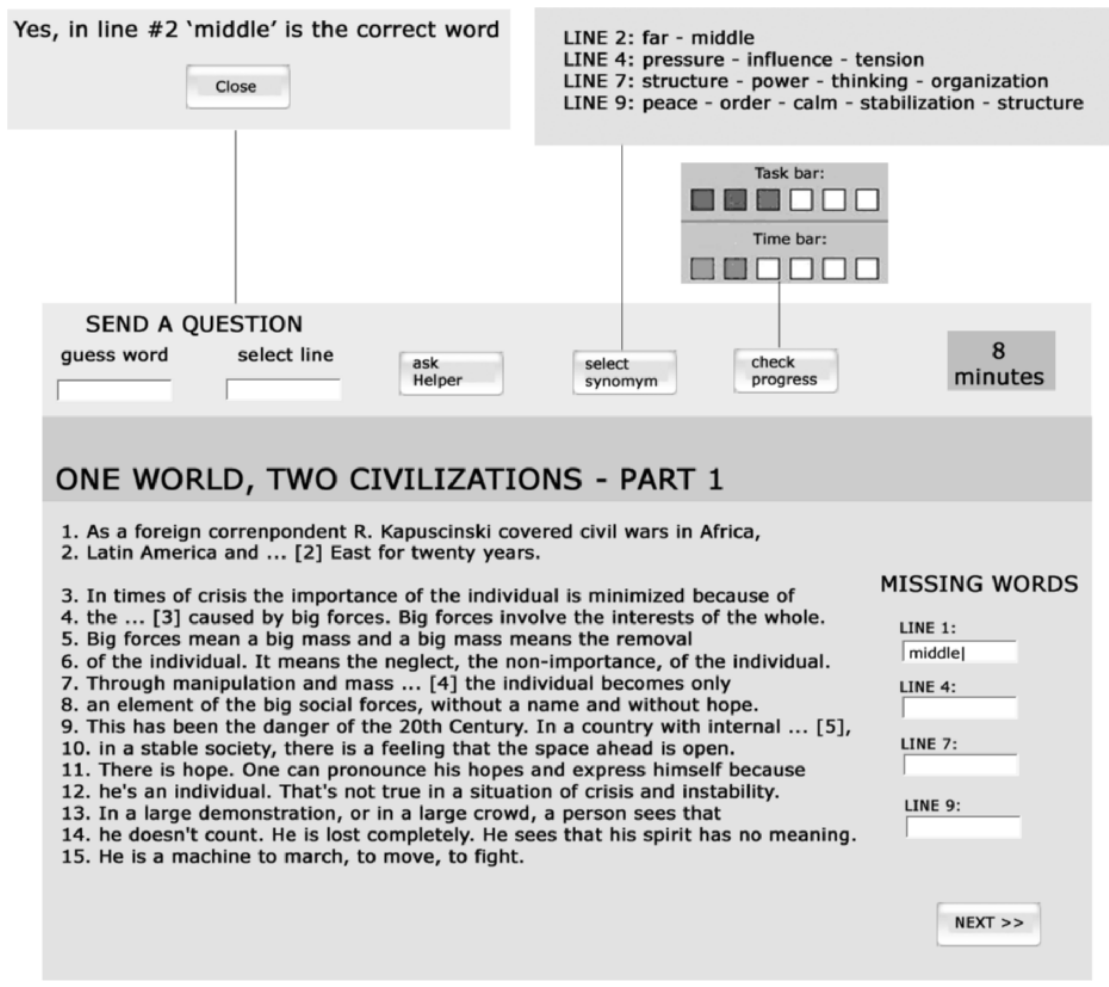

Figure 2. Asker's screen - the upper area contains of: $(1 \mathrm{a}, 1 \mathrm{~b})$ the form to ask questions and receive answers from the Helper, (2) the timer, (3) the button activating the awareness display and $(4 a, 4 b)$ the button with optional words to choose from; the lower area contains of: (5) the article and (6) the fields to enter missing words with a button to submit them.

\section{Helper's Game}

The Helper has to answer ten trivia questions by listing 6 related items (e.g., 'List six European capitals') and has 1 minute per question. Each consecutive answer scores more points (so the first answer scores 1 point and the sixth scores 6 points). After 1 minute a new question is displayed. 


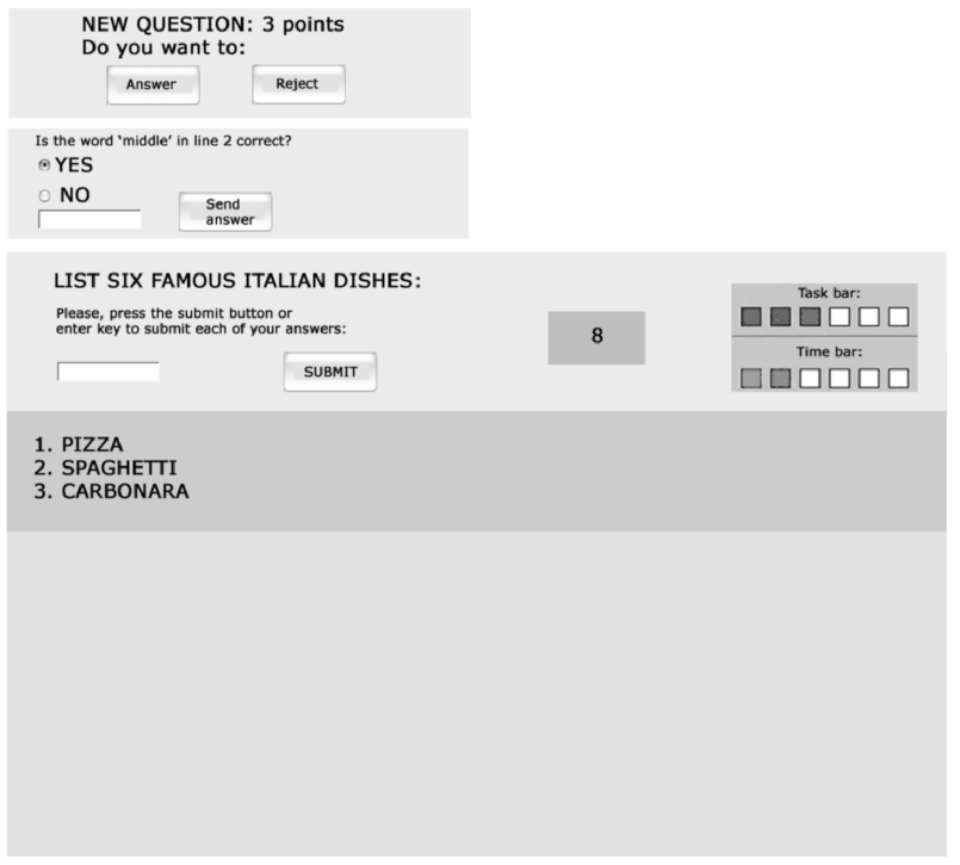

Figure 3. Helper's screen - the upper area contains of: (1) the question from the quiz, (2a) the notification of the new question from the Asker replacing the quiz question, (2b) the form to answer the Asker's question, (3) the timer and (4) the awareness display; the lower area contains (5) a list of answers submitted to the quiz question.

Figure 3 shows the Helper's screen that is also divided in two areas. The upper area displays the quiz questions and provides a text-field wherein the six answers should be entered, a timer and the awareness display representing Helper's own progress bar. In the lower area the list of submitted answers is displayed. When the Asker's question arrives, the upper area changes so that two buttons replace the quiz: 'Answer' and 'Reject'. If the Helper chooses to reject then the quiz is reactivated and the Asker receives an 'Ask later' reaction.

If the Helper decides to answer, the lower area of the screen is replaced by the same paragraph that the Asker sees with lines numbered and missing words marked in brackets. The upper area is replaced by the Asker's question (e.g., Is 'earth' the correct word for line 2?). The Helper can either answer 'Yes', if the selected word is correct or ' $N o$ ' is the word is incorrect. Optionally, (s)he can enter the correct word in the text field below, thus providing (with some extra effort) a high-value response. 


\section{Results}

The first hypothesis in this experiment predicts that players in the Team condition will tend to display altruistic interruption behaviour regardless the system they use. The second and third hypotheses assume that players in the Group condition will display altruistic interruption behaviour only if additional system filtering is added to shield interruptees from untimely interruptions. In Table II we provide an overview of the eight dependent variables concerning all examined interruption behaviours. We have clustered them according to whether they pertain to time or content criteria. It is important to note that for simplicity we only report the results regarding the altruistic behaviours. We do so because (i) a relatively small number of individualistic behaviours was found meaning that no significant differences between conditions were observed, and (ii) the individualistic behaviours followed a pattern consistent with our hypotheses, and reverse to the altruistic behaviours discussed below, so they do not add any extra insights to our discussion.

ASKERS

HELPERS

Dependent variables for time-related altruistic and individualistic behaviours

Timely interruption: interrupting when progress bars show task being equal or ahead of time.

Untimely interruption: interrupting when progress bars show time being ahead of task.
Timely reaction: accepting of the incoming interruption.

Untimely reaction: rejecting the incoming interruption.

Dependent variables for content-related altruistic and individualistic behaviours

High-value question: asking about a word that scores 4 and 5 points.

Low-value question: asking about a word that scores 2 or 3 points.
High-value response: providing the 'No' answer and the correct word if the Asker's guess was incorrect.

Low-value response: providing 'No' answer only if the Asker's guess was incorrect.

Table II. Dependent variables for Askers' and Helpers' time and content-related behaviours used in the experiment

\section{Testing the hypotheses}

The three hypotheses as stated in section 3.1 were tested using two-way mixed subjects' ANOVA, with two independent variables (1 within and 1 between subjects). We tested each hypothesis separately for Helpers and Askers, both for 
time-related and for content-related dependent variables. Figure 4 shows the graphical representation of the obtained results with four graphs each showing the number of altruistic behaviours in each given case. For example, the graph in the upper right hand shows the number of timely interruptions initiated by Askers, thus those interruptions that were initiated when the progress bar showed the task progression was equal or ahead of time progress.
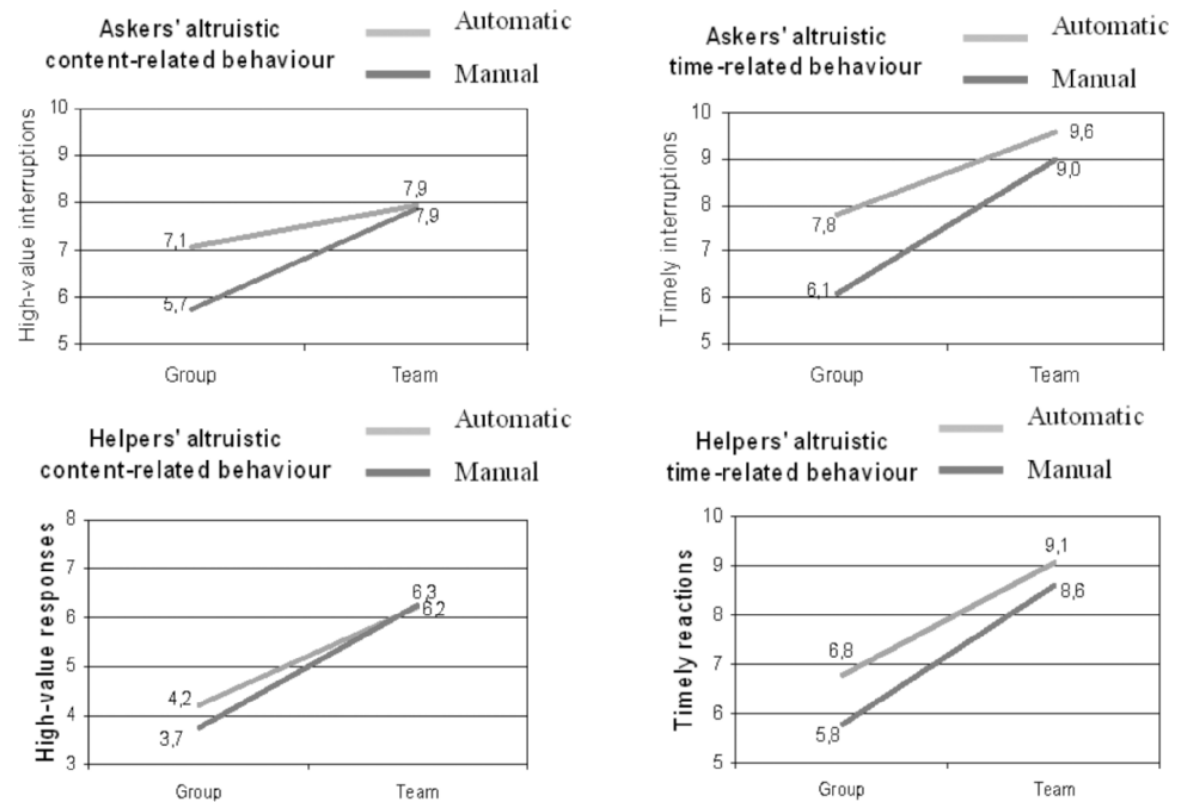

Figure 4. A graphical representation of the results of the quantitative analysis. Results are presented separately for Askers and Helpers, for both their time-related and their content-related behaviours.

Hypothesis 1 stated that in all cases, thus for all four dependent variables, players in the Team condition would show more altruistic behaviours than players in the Group condition. A graphical inspection of the data shows that for the altruistic measures presented in Figure 4 players in the Team condition indeed scored higher than players in the Group condition. This should result in a significant main effect of the social condition, which is not the case. However, the main effect of the social condition on Askers' timely behaviours is not significantly but indicative, $F(1,28)=3.228, p=0.083$. This is also true for the effect on Helpers' content-related behaviour, $F(1,28)=3,571, p=0.069$. Since the sample size of our experiment was relatively low to test this two-way model, we believe that a clearly visible overall trend in the graphical representation of the data confirm hypothesis 1 . 
Hypothesis 2 stated that players using the Automatic system would show more altruistic behaviours than players using the Manual system. From the graphs in Figure 4 it is visible that this is the case for all four of our dependent variables. For Askers' time-related behaviour the main effect of system is significant, $F(1$, $28)=4.388, p<0.05$; Askers in the Automatic system initiated on average 8.7 timely interruptions, while Askers in the Manual system initiated on average 7.5 timely interruptions. For the other three dependent variables the main effect of the system is not significant at 0.05 level. This lack of significance is again most probably due to the relatively low sample size of the experiment. Since a graphical inspection of the data clearly shows the main effect of the system type, we believe that we can validly accept hypothesis 2 or at least confirm that there is a clear indicative trend towards the positive effect of the Automatic system on peoples' interruption behaviours.

Hypothesis 3 stated that the positive effect of the system type, thus the higher number of altruistic behaviours in the Automatic system would be stronger in the Group condition. This hypothesis refers to an interaction effect between the system type and the social condition. Graphically this would result in converging or diverging lines in the graphs in Figure 4. In all four cases, the interaction effect is not significant. This experiment did not show that the effect of the system type was different for the different social conditions. Thus, we conclude that we have failed to find evidence to support hypothesis 3. In order to take a closer look into the collected data regarding the hypothesis 3 , we also analysed individual behavioural differences in the Team and Group conditions within each system. We ran independent samples T-Test to see whether the system shows an effect on altruistic behaviours separately for each social condition. It is interesting to mention a difference in the effect of the system on the altruistic behaviour of Askers in the Group condition that is not present in Team condition (Automatic-Group $M=$ 7.8; Manual-Group $M=6.07 ; t(14)=2.284, p<0.05$ ). However, the manipulations in our experiment were not strong enough to clearly show this interaction effect in the full two-way model. This result is by no means a sufficient evidence to support Hypothesis 3, however it opens a discussion about the potential influence of the system on behaviours in the group condition (see the discussion section).

\section{Qualitative results}

Statements (212) from six focus group sessions conducted with participants at the end of the experiment were audio recorded and transcribed with a notification whenever they were made by Askers or Helpers from either the Team or the Group condition. Next, passages describing participants' behaviours and motivations for each individual behaviour were extracted for further analysis (85 statements). These passages were coded by two independent coders according to 
whether they described participants' time or content-related behaviours and also according to comments about either the Manual or the Automatic system. Related statements within each group were clustered together, so that the differences between various motivations could be seen. The final step in the analysis was to reconnect the emerged clusters with data from the logs and examine how they relate to the quantitative results.

\section{Askers' motivations for different interruption behaviours}

Askers motivated initiating timely interruptions based on their individual and social concerns. The individual concern represented willingness to avoid rejection or to avoid waiting for Helper's answer. The social concern addressed willingness to avoid interrupting Helpers at wrong moments.

Team: "With the first (Automatic) system, you check progress bars more often because you want to ask the question only when it is useful to avoid rejection."

Team: "In this first (Manual) system I would check the progress bars and see if he had a lot of time left and then I would start asking."

Group: "I looked at the progress bars all the time, it has helped me to develop my strategy when to ask and have high chances not to be rejected."

Group: "... if the time bar were ahead of the task bar I would not ask any question. I was waiting a bit, checking again and if the situation didn't change, I would not bother him."

Interestingly, some Askers reported that the Automatic system relieved them of their social responsibility towards Helpers. They tended to check the progress bars less frequently and kept asking whenever necessary knowing that the system would notify them each time the interruption was untimely. In some cases, they checked the progress bars after interrupting to verify their chances for receiving a timely reaction. In the case of rejection, they would frequently perceive the 'Ask Later' reaction to be provided by the system and not the Helper (even if sometimes it was not the case).

Team: "But the second time (Automatic) I just asked whenever I felt like it because I knew that I would be rejected by the system if he was too busy. So, I let the system decide for me."

Group: "In the other (Automatic) system I kept on sending questions because I knew that if timing was wrong, the system would deal with it. And I didn't mind the system refusing me."

Askers motivated initiating high-quality interruptions as a way to improve their score. They often decided to guess low-quality questions themselves since the eventual loss of points was limited and they did not want to waste time waiting. They also tended to ask high-quality questions when they perceived highpressure in the Helper's game or if they wanted to avoid being too intrusive.

Team: "I didn't want to wait for easy answers. So, I just started with the difficult ones, which gained more points and then put the rest in, while I was waiting for the answers."

Group: "I got rejected on the third question and then I decided: I am not bothering him with any questions except from the most difficult ones". 


\section{Helpers' motivations for different interruption behaviours}

Helpers perceived that their availability was not well assessed either by the system or the Askers. They noticed that whenever they had ample time they would not receive interruptions, while when they were rather busy with their own game, interruptions would feel to be more frequent. In the Automatic system, Helpers reported feeling unable to help Askers at times when they got blocked on their own quiz game. Some Helpers started deceiving the system by putting bogus answers to simulate progress in the quiz. Nevertheless, when Helpers were performing well, they appreciated the protection of the Automatic system allowing them to first complete their own task and attend Asker's requests later.

Team: "At some point in the (Automatic) game I knew only three answers. So, I couldn't do anything anymore and I knew that the system wouldn't be sending any questions."

Team: "With the second (Automatic) system I [...] felt I can at least finish my thoughts and as soon as I am done I can help."

Group: "If I didn't know anything about the question, so I thought: 'I've already lost this one, I will at least help her'... So, I had more control over the game with the first (Manual) system than with the second (Automatic)."

Group: "With the second (Manual) system I felt I was getting more questions. With the other one (Automatic) the questions came when I had ample time to answer them."

For some Helpers high-quality questions had higher priority than their own quiz game, while low-quality questions had not.

Team: "With 5-point questions, I knew that no matter what I do, I could score only one extra point. So, it was definitely worth answering him."

Team: "At some point I got a question, which had only few points and I thought: I am going to gain more points with my answers than this one, so I rejected."

For others providing high-quality answers was a way to optimise their performance (not giving the right answer would increase chances of receiving the same question again) or a method to show the pressure of their own game. Providing high-quality answers were for some Helpers a way to balance their inability to help at all times.

Team: "I would check how many points the word was scoring. If it had more points than 2 then it was worthwhile for me to type in the whole thing, even if I missed my answer."

Team: "I thought it's just a waste of time to say 'No' only. Then you get the same question again $[\ldots]$ It is just easier to give the answer."

Group: "Then I answered the next questions putting the correct word but after that I felt more pressure and then I answered only 'No'."

\section{Discussion}

The quantitative results of our experiment provide a confirmation of hypothesis 1 showing that players in the Team condition (shared goal) presented more altruis- 
tic behaviours than players in the Group condition (non-shared goal). These results confirm findings of prior works and extend them by showing that interruptees display similar interruption behaviours to those presented by interruptors. Furthermore, our results suggest a positive effect of the Automatic system on peoples' interruption behaviour confirming hypothesis 2 . Finally, we could not find evidence to either accept or reject hypothesis 3 - there was no visible effect of the system type that over the two different social conditions.

Although we realize that the crudeness of the automation algorithm was a very simple scheme meant only to make sense in the experimental setup our results in fact represent a broader context, which we would like to elaborate further on. Specifically we would like to explain the outcome of Hypothesis 2 based on our qualitative findings. Social interactions are dynamic and, even in our case where static team-group relationships was defined between the interruptor and the interruptee, the two actors did not always act in an equally straightforward way as a team or as a group. Looking closer into the effects of the Automatic system we consider it as a system protecting the interruptees and at the same time punishing the interruptors for improper timing of their interruptions. We use the notions of protection and punishment to translate our results into design implications. We argue that these effects put to an individual level the costs associated with different interruption behaviours. We cannot conclude that an Automatic system lead people to feel more considerate towards their partners. Nonetheless, we believe that participants behaved in a less individualistic manner mainly because they perceived the individual costs of their actions as more consequential comparing to their costs in the Manual system.

Askers associated costs with the way the Automatic system handled interruptions, namely that untimely interruptions were automatically rejected. As hypothesized, the Automatic system did not impact the behaviour of Team-Askers, who timed their interruptions well. On the other hand, it forced Group-Askers to be more conscious about timing their interruptions as a way to reduce the rejection ratio and to minimize time spent waiting for the Helper's response. At the same time the Automatic system made Askers feel relaxed to interrupt at any moment based on the knowledge that whenever they chose the interruption moment badly, the system would reject them automatically and the Helper would be in no way affected by their poor choice. Moreover, no costs were clearly associated with providing a wrong answer in the Askers' game, thus in both social conditions Askers showed to be little motivated to initiate interruptions that could bring low-value for either them individually or for the team and to be highly motivated to limit interruptions to only those with high score. A question, however, remains what would have happened if there was a cost associated with giving a wrong answer and how such a cost would have affected Askers' behaviours in both social conditions. 
Similarly, Helpers saw the costs of providing low-value responses because of the high probability of being interrupted with the same question again. Once Helpers in both social conditions had decided to accept the interruption they tended to provide a comprehensive rather than a parsimonious response.

Interestingly, Helpers' preference between the two systems depended on their individual performance. They preferred the Automatic system whenever they performed their own task well; otherwise they preferred the Manual system allowing them to use time, which they would otherwise waste, for helping Askers.

These conclusions lead to design implications built on the notions of: interruption cost, social tension and system preference (see: Table III). Interruptees have demonstrated the need to modify the system behaviour in accordance to their performance and so it allows them to switch from synchronous to asynchronous communication whenever necessary. The chosen system behaviour should, however, be clearly indicated to both interacting parties, so interruptors would remain aware of the interruptees' choice and could adapt their expectations and behaviours accordingly. However, once the interruptee chooses for the automatic filtering, switching to the asynchronous communication, the system should provide interruptors with a buffer, in which they could store the content of their interruptions. Such a buffer would allow releasing the social tension guaranteeing to the interruptor that the interruption would reach the interruptee at an appropriate moment.

\begin{tabular}{l|l|l}
\multicolumn{1}{c|}{ Interruption Costs } & \multicolumn{1}{c|}{ Social Tension } & \multicolumn{1}{c}{ System Preference } \\
\hline $\begin{array}{l}\text { Interruptees try to avoid } \\
\text { being interrupted with the } \\
\text { same content again. }\end{array}$ & $\begin{array}{l}\text { Interruptors shift the re- } \\
\text { sponsibility of assessing } \\
\text { the appropriate interrup- } \\
\text { tion moment to the sys- }\end{array}$ & $\begin{array}{l}\text { Interruptees prefer addi- } \\
\text { tional system protection } \\
\text { only when they perceive } \\
\text { that their own task is worth } \\
\text { to continue working on. }\end{array}$ \\
$\begin{array}{l}\text { Interruptors try to avoid } \\
\text { being idle waiting for an } \\
\text { inconclusive response. }\end{array}$ & tem. & \\
\hline
\end{tabular}

Design Implications

Provide mechanisms to assign individual costs when one behaves socially inappropriate.

Provide indicators to make these costs visible.
Provide a buffer to queue untimely interruptions.

Allow interruptee to access interruptions in the buffer at any moment.
Design an availability communicator, where actors indicate whether they would like to coordinate interruptions manually or they prefer automatic interruption filtering.

Table III. Design implications built on the notion of interruption costs, social tension and system preference 


\section{Conclusions}

We have presented an experimental study of interruption behaviours, in which we have compared an automatic versus a manual approach to handle interruptions. Our findings show that the automatic system encouraged altruistic behaviours more than the manual system. We have also compared the behaviour of interruption actors who share a common goal, versus those whom only dependency is potential reciprocation. Consistently with prior works our results indicate that altruistic behaviours are shown by interruptors who share a common goal with interruptees. We have also measured that interruptees presented similar behavioural patterns as interruptors. We did not find differences in the impact of the system type for the two social relationships.

Based on the qualitative analysis that tries to explain the results of this experiment, particularly of Hypothesis 2, we have deduced a number of design implications. Evidences show that the behaviour of the system depends on moment-tomoment activities of the two actors, suggesting the need for an adaptable interruption handling strategy. As an interruption brings individual costs to both actors a clear indication of these costs should be displayed to them. Consistent with the first conclusion the chosen strategy should be clearly observable by both actors to evaluate the potential costs of the interruption.

In the next steps of this research, we shall seek to verify the suggested design implications by applying them in the design of technologies to support interruption handling of collocated collaborators.

\section{References}

Adamczyk, P. D. and B. P. Bailey (2004). 'If not now, when?: the effects of interruption at different moments within task execution'. CHI, ACM Press.

Altman, I. (1975). The Environment and Social Behaviour - Privacy, personal space, territory, crowding. Monterey $(\mathrm{Ca})$, Wadsworth

Bailey, B. P., J. A. Konstan, et al. (2005). 'On the Need for Attention-Aware Systems: Effects of Interruption on Task Performance, Error Rate, and Affective State.' Journal of Computers in Human Behavior 1 (special issue on attention aware systems)

Begole, J. B., N. E. Matsakis, et al. (2004). 'Lilsys: Sensing Unavailability'. CSCW, ACM Press

Begole, J. B., J. C. Tang, et al. (2003). 'Rhythm modelling, visualizations and applications.' UIST, ACM Press

Cheverst, K., A. Dix, et al. (2005). 'Exploring bluetooth based mobile phone interaction with the hermes photo display.' Mobile HCI, ACM Press

Chrysanthis, P. K., D. Stemple, et al. (1990).' A logically distributed approach for structuring office systems.' SIGGROUP, ACM Press,

Clark, H. (1996). Using language. New York, Cambridge University Press

Dabbish, L. and R. Kraut (2004). 'Controlling Interruptions: Awareness Displays and Social Motivation for Coordination.' CSCW, ACM Press 
Dourish, P. and S. Bly (1992). 'Portholes: supporting awareness in a distributed work group.' CHI, ACM Press

Fogarty, J., S. E. Hudson, et al. (2005). 'Predicting human interruptability with sensors.' $A C M$ Transactions on Computer-Human Interaction vol. 12, no. 1, pp. 119-146

Goffman, E. (1967). Interaction Ritual: Essays in Face-to-face Behavior. Random House Inc.

Gonzales, V. M. and G. Mark (2005). 'Managing currents of work: Multi-tasking among multiple collaborations.' CSCW, Springer

González, V. M. and G. Mark (2004). 'Constant, constant, multi-tasking craziness.' CHI, ACM Press

Hudson, J. M., J. Christensen, et al. (2002). 'I'd be overwhelmed, but it's just one more thing to do.' CHI, ACM Press

Jett, Q. R. and J. M. George (2003). 'Work interrupted: A closer look at the role of interruptions in organizational life.' Academy-of-Management-Review vol. 28, no. 3, pp. 494-507

Kakihara M., Sorensen C. and Wiberg M. (2004). 'Fluid interaction in mobile work practices, The Interaction Society: Practice, Theories, and Supportive Technologies', The Interaction Society: Practice, Theories and Supportive Technologies, IDEA-group Inc.

Kendon, A. (1990). Conducting interaction: patterns of behavior in focused encounters, Cambridge University Press

Kraut, R. E., R. S. Fish, et al. (1990). 'Informal communication in organizations: Form, function, and technology.' Human reactions to technology., Claremont symposium on applied social psychology, Beverly Hills, CA, Sage Publications

McFarlane, D. C. (2002). 'Comparison of Four Primary Methods for Coordinating the Interruption of People in Human-Computer Interaction.' HUMAN COMPUTER INTERACTION HILLSDALE THEN MAHWAH vol. 17, no. 1, pp. 63-139

McFarlane, D. C. and K. A. Latorella (2002). 'The Scope and Importance of Human Interruption in Human-Computer Interaction Design.' Human-Computer Interaction vol. 17, no. 1, pp. 161,2002

Minassian, S. O., M. J. Muller, et al. (2004). Diverse Strategies for Interruption Management in Complex Office Activities.' IBM Watson Research Center

Nardi, B. and S. Whittaker (2001). 'The place of face-to-face communication in distributed work.' Distributed Work. P. Hinds and S. Kiesler, MIT Press

Nardi, B. A., S. Whittaker, et al. (2000). 'Interaction and outeraction: instant messaging in action.' $C S C W$, ACM Press

Nichols, J., J. O. Wobbrock, et al. (2002). 'Mediator and medium: doors as interruption gateways and aesthetic displays.' DIS, ACM Press

Olson, G. M. and J. S. Olson (2000). 'Distance Matters.' Human Computer Interaction -Hillsdale Then Mahwah vol. 15, no. 2/3, pp. 139-178

Patil, S. and J. Lai (2005). 'Who gets to know what when: configuring privacy permissions in an awareness application.' $\mathrm{CHI}$, ACM Press

Perlow, L. and J. Weeks (2002). 'Who's Helping Whom? Layers of Culture and Workplace Behavior.' Journal on Organizational Behaviour vol. 23, pp. 345-362

Reichenbach, M., H. Damker, et al. (1997). 'Individual management of personal reachability in mobile communication.' SEC, IFIP TC

Sproull, L. S. (1984). 'The Nature of managerial Attention. Advances in Information Processing in Organizations.' JAI Press

Wiberg, M. and S. Whittaker (2005). 'Managing availability: supporting lightweight negotiations to handle interruptions.' ACM Transactions on Computer Human Interaction vol. 1 , no. 12 